\title{
Modeling GW Generation at Start of the Electro Weak Regime and Its Tie into the Machian Universe with Falsifiable $h_{i j}$ Values
}

\author{
Andrew Walcott Beckwith \\ Physics Department, College of Physics, Chongqing University Huxi Campus, Chongqing, China \\ Email: Rwill9955b@gmail.com, abeckwith@uh.edu
}

How to cite this paper: Beckwith, A.W. (2017) Modeling GW Generation at Start of the Electro Weak Regime and Its Tie into the Machian Universe with Falsifiable $h_{i j}$ Values. Journal of High Energy Physics, Gravitation and Cosmology, 3, 308-321. https://doi.org/10.4236/jhepgc.2017.32026

Received: January 18, 2016

Accepted: April 17, 2017

Published: April 20, 2017

Copyright $\odot 2017$ by author and Scientific Research Publishing Inc. This work is licensed under the Creative Commons Attribution International License (CC BY 4.0).

http://creativecommons.org/licenses/by/4.0/

cc) (i) Open Access

\section{Abstract}

The early electro weak regime as of $10^{-32}$ seconds after the big bang is where we could see the initial formation of gravitons, gravitinos and $G W$. What we intend to do is to look at if Mach's principle, and a statement of overall quantized energy state behavior of the universe can help us get $h_{i j}$, using initial conditions as initially presented by Mishra in 2012 we restate as $\frac{G M}{R_{0} c^{2}} \approx$ const . Mach's principle was used by Mishra, and we use it to come up with conditions for a stable overall mass $M$ contributing to $G W$ generation/ entropy of the universe. The composition of $M$ for gravitons would change over time from initial beginnings to the present day, but the final invariant graviton mass $M$ we work with is a way to state initial and final numbers, $N$, of the constituent particles contributing to entropy of our universe. By the way of comparison this also is tied into Gravitinos, as super partners to Gravitons, as counted by $N$, initially, and dying out as up to the present day values. From the present, we have the Machian condition of setting, the present condition, as given by Mishra $\frac{G M}{R_{0} c^{2}} \approx$ const, with $M=N m$ being the mass of a sub-system inside the universe, with $N$ being the number of "particles", and $m$ being the net particle mass. We examine the consequences of Mach's principle for the case of the mass $M$, contributing to $G W$ and entropy with a case of $M_{\text {electro-weak }} \approx M_{\text {today }}$, i.e. the total mass of the electro weak era is about the same as today's mass, but if we look directly at the influence of SUSY physics super partners, in such a way that

$\left.M_{\text {electro-weak }}\right|_{\text {Super-partner }} \neq\left. M_{\text {electro-weak }}\right|_{\text {Not-Super-Partner }}$ and there is then an equivalence between SUSY dominated early conditions and non-SUSY 
$\frac{\left.G M_{\text {electro-weak }}\right|_{\text {Super-partner }}}{R_{\text {electro-weak }} c^{2}} \approx \frac{\left.G M_{\text {today }}\right|_{\text {Not-Super-Partner }}}{R_{0} c^{2}}$ as equal to a constant value. i.e. if Machian physics held from early times, up to the present, it would have implications for explaining entropy, as given in

$\left.S_{\text {entropy }}\right|_{N g-Q M-\infty-\text {-statistics }} \sim N_{\text {Particle-count }}$, as to why it would be so much lower as of about and before the electro-weak regime than today. This leads to Equations (37)-(39) as $h_{i j}$ values to be detected by appropriate GW detectors.

\section{Keywords}

Gravitons, Gravitinos, Entropy, Machian Universe, Electro-Weak, SUSY Physics, Super Partners

\section{Introduction: Ranges of Masses Considered and Lifetimes}

We give support to the idea that a Gravitino would (as a much more massive particle than even a massive graviton) have a far shorter life time than a graviton, even in the case of Massive gravitons. This will have implications in terms of applying Mach's principle, which is done in this document.

We will work with a seemingly naive interpretation of looking at gravitons, and gravitinos, as given by Sarkar [1] the mass of a rest Gravitino would be for a temperature about $10^{12} \mathrm{~K} \leq T_{\text {inf }} \leq 10^{12+\beta} K$ up to $10^{-32}$ seconds

$$
1 \mathrm{TeV}<m_{3 / 2} \equiv m_{\text {Gravitino }}<5 \mathrm{TeV}
$$

Note that gravitinos have a very short life time, and KORI et al. [2] state that the lifetime of the Gravitino goes down as its mass goes up, i.e.

$$
\tau_{\text {Gravitino }}=\text { Life }- \text { time }=1 / \Gamma\left(\psi_{3 / 2} \rightarrow \text { all }\right)
$$

On page 10, Kori et al. [2] have that $\tau_{\text {Gravitino }}=$ Life - time $=1 / \Gamma\left(\psi_{3 / 2} \rightarrow\right.$ all $)$ $<10^{4} \mathrm{sec}$ with frequent values for the Gravitino life time down to as low as 1 second, i.e. not lasting long in the neighborhood of the electro weak regime. The electro weak phase would be for 0.3 meters in diameter according to Giovanni [3] in a California institute of technology website, and graviton production as well as gravitino production would start as early as $10^{-35}-10^{-32}$ seconds according to [1] and also confirmed by a university of Oregon Physics website [4].

Note that in the vicinity of the electro weak regime, the magnetic field was probably enormous, i.e. the obtained magnetic fields were rather strong (i.e. $|\boldsymbol{B}| \sim 10^{22} \mathrm{G}$ at the EW epoch) [3] but over a small scale, i.e. $10^{-6} H_{e w}^{-1}$.

The lime time of a graviton if it is a spin two-zero mass boson is effectively infinite. For $K K$ gravitons, as given by Sarkar [1], the way to get the life time is to make use of, and for mode $m_{n}$ is to look at

$$
\tau_{K K \text {-Graviton }} \sim 10^{31} \text { years } \cdot\left[\frac{1 \mathrm{eV}}{m_{n}}\right]^{3}
$$

If one is not using $K K$ gravitons, but assuming massive gravitons, then [5] 
gives the hint, in stating "in conclusion, only the complete non perturbative quantum theory can fix the lifetime of the graviton".

If we made the substitution

$$
\begin{aligned}
& m_{n} \rightarrow m_{\text {graviton }} \sim 10^{-26} \mathrm{eV} \\
& m_{n} \rightarrow m_{\text {graviton }} \sim 10^{-26} \mathrm{eV}
\end{aligned}
$$

A good non-perturbative effect may be to go to Equation (3) and to look at $m_{n}$ as for a graviton life time [1]

$$
\tau_{K K \text { Graviton }} \sim 10^{31} \text { years } \cdot\left[\frac{1 \mathrm{eV}}{m_{n}}\right]^{3} \rightarrow 10^{31} \text { years } \cdot\left[\frac{1 \mathrm{eV}}{10^{-26} \mathrm{eV}}\right]^{3} \sim 10^{57} \text { years }
$$

Usually the lifetime of the universe is considered to be $3.4 \times 10^{10}$ years [6] and (Big rip scenario) of Equation (5) is considerably longer than the expected lifetime of the universe, which is not surprising.

Applying the Machian principle to Gravitinos at $10^{-32}$ seconds, and a 0.3 meter radius, of a universe, versus a present universe radius of about $1.422 \times 10^{27}$ meters after $4.41796 \times 10^{17}$ seconds [6], i.e. a difference in radius we can write as

$$
\frac{R_{0}}{R_{\text {Electro-Weak }}}=\frac{1.422 \times 10^{27}}{3 \times 10^{-1}} \sim .47 \times 10^{28}
$$

Now let us make the following assumption. That for each graviton, there is a counterpart Gravitino in the electro weak regime, i.e. up to a point we have the following, and i.e. an early universe version of Mach's principle as to Gravitons we can expression as follows

$$
\begin{aligned}
\frac{\left.G M_{\text {electro-weak }}\right|_{\text {Super-partner }}}{R_{\text {electro-weak }} c^{2}} & \approx \frac{0.47 \times 10^{28} \times\left. G M_{\text {electro-weak }}\right|_{\text {Super-partner }}}{R_{0} c^{2}} \\
& \approx \frac{\left.G M_{\text {today }}\right|_{\text {Not-Super-Partner }}}{R_{0} c^{2}} \approx \text { const }
\end{aligned}
$$

This implies then,

$$
0.47 \times 10^{28} \times\left.\left. M_{\text {electro-weak }}\right|_{\text {Super-partner }} \approx M_{\text {today }}\right|_{\text {Not-Super-Partner }}
$$

If so, then the number of super partner Gravitons equals the number of gravitinos in the Electro weak era, and one has

$$
M_{\text {electro-weak }}=N_{\text {electro-weak }} \cdot m_{3 / 2}=N_{\text {electro-weak }} \times 10^{38} \cdot m_{\text {graviton }}
$$

So

$$
\begin{aligned}
M_{\text {electro-weak }} & =N_{\text {electro-weak }} \cdot m_{3 / 2}=N_{\text {electro-weak }} \times 10^{38} \cdot m_{\text {graviton }} \\
& =N_{\text {today }} \cdot m_{\text {graviton }} \approx 10^{88} \cdot m_{\text {graviton }}
\end{aligned}
$$

Then the electro weak regime would have

$$
N_{\text {electro-weak }} \sim 10^{50}
$$

Using quantum infinite statistics, this is a way of fixing the early electro weak 
entropy as $\sim 10^{50}$ vs. $10^{88}$ today, i.e. this uses $\mathrm{Ng}^{\prime}$ 's quantum infinite statistics, to get $S \sim N$.

\section{We Have Concluded a Proof, That Entropy, according to Mach's Principle Grows $1^{38}$ Times from the Electro Weak Era. From $\sim 10^{50}$ to $10^{88}$, What Else? How Do We Get to Have the Entropy Figure of $10^{50}$ ?}

Note that for a $K K$ graviton that there is a mass, which we can call as follows, traditionally one has, then

$$
m_{n}^{2} \sim \mu^{2}+\frac{n^{2}}{r^{2}} \underset{\mu \rightarrow \varepsilon^{+}}{\longrightarrow} \frac{n^{2}}{r^{2}}+\varepsilon^{+} \approx \frac{n^{2}}{r^{2}}
$$

Note that as of the Planck scale we would be working with the following. Start off with Planck mass, with $M_{\text {Planck }} \sim \frac{1}{\sqrt{G_{N}}} \sim 10^{19} \mathrm{GeV}$, then Planck length $L_{\text {Planck }} \sim \sqrt{G_{N}} \sim 10^{-33} \mathrm{~cm}=10^{-34} \mathrm{~m}$ and also Planck time as $t_{\text {Planck }} \sim 10^{-44} \mathrm{sec}$

The question we can ask is then, what would a spatial distance would correspond to a graviton mass, the surprising answer is

$$
1 / \widehat{r} \sim m_{\text {graviton }} \propto 10^{-26} \mathrm{eV} \Rightarrow \widehat{r} \sim 10^{20} \mathrm{~m}
$$

Note that the radii of the present universe, in four dimensions is usually thought to be of the order of, as given by Mishra [6] of the value $R_{0} \sim 10^{27}$ meters, so then the

$$
R_{0} \sim 10^{27} \text { meters } \approx 10^{7} \hat{r}
$$

Here a nonstandard version of $K K$ theory is that in space time, one usually thinks of higher dimensions as of Planck sized spatial contributions, and Arkani Hamid [7] [8] still was very conservative in this matter.

But if there is a prior universe, and that due to cyclic conformal cosmology [9], and a Meta structure containing the 4 dimensional structure, then $\widehat{r} \sim 10^{20} \mathrm{~m}$ as far as an embedding structure is not so fantastic after all. Using Penrose's formulation from his 2010 book [9], we will report on vacuum energy and its connections to entropy. The rub in all of this though is that Penrose never explained how to go from his cyclic conformal cosmology collection of matter from a million or so black holes, to a new universe, a process with initially low temperatures (Black holes eventually evaporate) to the higher temperatures associated with a new big bang. We provide such a driver via use of an addition of the Einstein energy stress tensor with an electromagnetic addition to it [9]

$$
\begin{aligned}
& \left.\Lambda\right|_{\text {initial-Planck time }} \text { [vacuum - energy] } \\
& \sim \frac{c^{3}}{N^{6} G \hbar} \approx 10^{-6}-10^{-24} \\
& \sim 1 / S_{\text {initial-Planck-time }}
\end{aligned}
$$

Take in mind that the above Equation (15) is assuming a Quintessence set of conditions, i.e. that the vacuum space time changes from initial conditions to the Electro weak era and then to today. The question though is when the electro 
weak era would actually begin. We can reference a treatment of Hubble time, as follows with the Hubble parameter set, Sarkar [1], as to early universe Hubble parameters

$$
H_{\text {Early-Universe }} \sim 1.66 \times \sqrt{g^{*}} \times \frac{T_{\text {Early-Universe }}}{M_{\text {mass-scale }}}
$$

The electro weak regime, depending upon the evolving values of $g^{*}$ could vary between $10^{-35}$ seconds to as "large "as $10^{-32}$ seconds.

Making use of what was done by Beckwith [10] at DICE, 2010, as to $g^{*}$ rising to at or above 1000, instead of the commonly accepted figure of 100 or so given by Kolb and Turner in 1991 [11], as a chaotic map driven increase in degrees of freedom from a low point to a high point. With vacuum thermal energy initially tied to [10]

$$
g_{i+1}^{*}=\exp \left[-\alpha g_{i}^{*}\right]+\left[(\text { vacuum }- \text { thermal - energy })_{i}\right]
$$

The vacuum thermal energy in this case given by quiescent behavior in the vicinity of the electro weak regime would be given by [12] and subsequently modified by Beckwith

$$
\begin{aligned}
& \Lambda[\text { Park, 2002 }] \equiv \\
& \Lambda_{\text {General-Value }} T_{\text {Temperature }}^{\beta} \sim \frac{10^{-120} \cdot T_{\text {Temperature }}^{\beta=15 / 31}}{[2.73 \text { Kelvin }]}
\end{aligned}
$$

The upshot in terms of entropy would be a vacuum energy evolving as follows. Namely [12]

$$
\begin{aligned}
& \Lambda\left[\text { Park, 2002] }\left.\right|_{T_{\text {temp }}=10^{32} \text { Kelvin }} \sim 10^{-5} \propto \frac{1}{S_{\text {initial }}}\right. \\
& \Lambda\left[\text { Park, 2002] }\left.\right|_{T_{\text {teday }}=2.73 \text { Kelvin }} \sim 10^{-120}-10^{-124} \propto \frac{1}{S_{\text {Today }}}\right.
\end{aligned}
$$

There are two questions this raises. What would be the driving impetus to go from a low temperature pre space time temperature, then to Planck time entropy, then to the entropy of today as given in Equation (19)? This is similar to what would lead to the Electro weak era behavior, as far as an increase to the degrees of freedom. The way to do it would be to have an energy "driver" of inflation. One way to look at it would be to suggest that as done by H. Kadlecova [13] in the 12 Marcel Grossman meeting that the typical energy stress tensor, using, instead, Gyratons, with an electro-magnetic energy density addition to effective Electromagnetic cosmological value as given by

$$
\rho_{E \& M-\text { contribution }} \sim 8 \pi G \cdot\left(E^{2}+B^{2}\right)
$$

i.e. that there be, due to effective $E$ and $M$ fields a boost from an initially low vacuum energy to a higher ones, as given by Kadlecova [13] [14]

$$
\Lambda_{+}=\Lambda+\rho_{E \& M-\text { contribution }}
$$

Using the principle that one's E field is really another man's $B$ field, and a magnetic field of about $|\boldsymbol{B}| \sim 10^{22} \mathrm{G}$ at the pre-planckian to $E W$ epoch, we 
could then have a low temperature initial starting point for pre Planckian physics and then by both Equation (19) to Equation (20) go to dramatically increased temperatures, while leading eventually to conditions of Electro Weak space time physics which would be predicted by $\mathrm{Ng}$ infinite quantum statistics, which was given by $\mathrm{Ng}$ at the 12 Marcel Grossman conference [15], after a phase transition to the form of a perfect "graviton" gas looking with initial volume

$$
V_{\text {electro-weak }} \sim R_{H(\text { (electro-weak })}^{3}
$$

With a temperature of the order of $10^{12} K \leq T_{\text {inf }} \leq 10^{12+\beta} K$, for a net contribution of temperature due to

$$
T_{\text {Electro-weak }} \sim R_{H(\text { electro-weak })}^{-1} \propto \lambda_{\text {Electro-weak }}^{-1}
$$

And a numerical count we can give as

$$
N_{\text {electro-weak }} \sim\left[R_{H(\text { electro-weak) }} / l_{\text {Planck }} \propto 10^{25}\right]^{2} \equiv 10^{49}-10^{50}
$$

Note that $\mathrm{Ng}$ [15] [16], also has that the Hubble radius leading to an effective contribution due to the Electro Weak regime which would be about $0.3-0.4$ meters in length

$$
R_{H} \propto H^{-1} \Rightarrow R_{H(\text { Electro-Weak })} \propto H_{(\text {Electro-Weak })}^{-1}
$$

We submit that the Electro weak regime, will be where Gravitinos form, as of having mass of about $1 \mathrm{TeV}$, with $10^{50} \mathrm{TeV} \sim$ net mass $M$ contributing to $G W$ from the electro weak regime which will be part of the $h_{i j}$ calculations in the next section. About $10^{50} \mathrm{TeV}=10^{23}$ grams . The universe has a "mass" quantified regime of much greater value of, according to Mishra [6] $10^{56}$ grams, with the following conservation law, of sorts to be worked with as far as information, namely for preserving the cosmological constant information, we would have

$$
N_{\text {Today }} \times m_{\text {graviton }} \equiv 10^{88} \times m_{\text {graviton }}=N_{\text {electro-weak }} \times m_{\text {gravitino }} \equiv 10^{50} \times m_{\text {gravitino }}
$$

Next, note that as given by Giovanni, the figure of $10^{88}$ as due to gravitons can be seen to come from [17], page 156 as

$$
\begin{aligned}
& S_{\text {Graviton-Today's.ra }} \propto V \int_{10^{-19} \mathrm{Hertz}}^{10^{11} \mathrm{Hertz}} r(v) \cdot v^{2} \mathrm{~d} v \\
& \cong\left(10^{30}\right)^{3} \times\left(\frac{H_{1}}{M_{\text {Planck-mass }}}\right) \propto 10^{88}-10^{90} \\
& \sim\left(\text { difference of } 10^{11} \text { to } 10^{-19} \mathrm{Hertz}\right)^{3}
\end{aligned}
$$

\section{Review of the Penrose Cyclic Cosmology Conjecture in 4 Dimensions, Plus What Can Be Said about Black Holes and Fifth Dimensions, Etc.}

As given by Penrose [9], the phase space for gravitons in four dimensions can be seen to be

$10^{10^{90}} \approx 10^{\text {Final-entropy-Gravitons }=S_{\text {FINAL-GRAVIToNS }}}$

$\sim$ Phase space due to Gravitons 
The Penrose conjecture is that there is no big crunch that the universe continues to expand, with matter-energy trapped in black holes. Our hypothesis is that Black holes are actually 5 dimensional space time entities, i.e. then look at a much bigger phase space for containment of our 4 dimensional universe [9]

$$
\begin{aligned}
& 10^{10^{124}} \approx 10^{\text {Final-entropy-Galactic-Black-Holes }=S_{\text {FINAL-BL }}} \\
& \sim \text { Phase space Super - Massive }-B H-\text { in }- \text { Galaxies }
\end{aligned}
$$

The matter-energy trapped in black holes is assumed to be conformally mapped back in cyclic conformal cosmology to a new big bang, in so many words, gravitational "energy" is collected and re cycled. This is what Penrose wrote: From page 130 of his reference. Namely look at [9]

$$
\begin{aligned}
& E=8 \pi \cdot T+\Lambda \cdot g \\
& E=\text { source for gravitational field } \\
& T=\text { mass energy density } \\
& g=\text { gravitational metric } \\
& \Lambda=\text { vacuum energy, rescaled as follows } \\
& g \underset{c c c}{\longrightarrow} \hat{g}=\Omega^{2} g
\end{aligned}
$$

Note that the idea is conformal invariance, and this is similar to what is done in electromagnetism, as seen by Penrose's [9]

$$
\begin{aligned}
& \nabla F=4 \pi J \\
& F=\text { Field } \\
& J=\text { Current } \underset{\text { set-to }}{\longrightarrow} 0
\end{aligned}
$$

For cyclic conformal cosmology the basic construction is as follows. Namely look at

- Set a 'field' as $\phi_{A B C . . E}$

- Then the following holds. True for almost massless fields as well (i.e. the ultra-light graviton)

$$
\begin{aligned}
& \hat{\phi}_{A B C . . E}=\Omega^{-1} \phi_{A B C . . E} \\
& \hat{\nabla}^{A A^{\prime}} \hat{\phi}_{A B C . . E}=\Omega^{-3} \nabla^{A A^{\prime}} \phi_{A B C . . E} \\
& \text { Ideally, we have } \hat{\nabla} \hat{F}=0 \Leftrightarrow \nabla F=0
\end{aligned}
$$

For CCC theory, Penrose (2010) makes the following mapping.

$$
\Omega \underset{C C C}{\longrightarrow} \Omega^{-1}
$$

The cross over from Cycle to Cycle is given by mapping in Equation (33) above. And the invariance, as in Equation (32)

$$
\begin{aligned}
& \hat{K}=\Omega^{-1} \hat{C} \\
& \hat{\nabla} \hat{K}=0,
\end{aligned}
$$

and before crossover to new universe zone

$C \rightarrow \varepsilon^{+} \approx 0^{+}$just before crossover,

$\Omega \rightarrow$ tiny value

$K$ remains finite

$$
\Pi=\frac{d \Omega}{\Omega^{2}-1} \underset{\Omega \underset{C C C}{\longrightarrow} \Omega^{-1}}{\longrightarrow} \operatorname{SAME} \frac{d \Omega}{\Omega^{2}-1}
$$


Key hypothesis in this presentation, i.e. a graviton can obtain effective mass in the regime before the start of a new mapping. i.e. one can have no issues as to forming a fifth dimensional value of $\hat{r} \sim 10^{20} \mathrm{~m}$ as far as an embedding structure for the first tier of a $K K$ graviton is not so fantastic after all. This would be shoe horned into the four dimensional space time continuum, and be carried through to the electro weak regime, and each graviton super partnered with a gravitino.

\section{Shifting the Pre $C C C$ Regime First KK Mass for a Graviton to the Zeroth Order KK Mode, Giving a Graviton a Tiny Effective Mass}

Hypothesis. Pre CCC has a $1 / r 1^{\text {st }}$ excited $K K$ state for the graviton with $\hat{r} \sim 10^{20} \mathrm{~m}$ which then gets shifted to the zeroth order mass in the following formulation. So, let us review the idea of a MASSIVE graviton in terms of $K K$ theory.

We would get, then. as was given in Beckwith's 2011 Journal of Cosmology article [18], assuming that the prior $c c c$ cycle first $K K$ spatial radius $r$ was huge in pre cyclic conformal cosmology, and then the $1 / r$ value shifted to a zeroth order mode contribution of [18] and making use of Sarkar [1] as well, i.e.

$$
\begin{aligned}
m_{n}(\text { Graviton }) & =\sqrt{\frac{n^{2}}{L^{2}}+\left(m_{\text {graviton-rest-mass }}=10^{-65} \text { grams }\right)^{2}} \\
& =\frac{n}{L}+10^{-65} \text { grams }
\end{aligned}
$$

The zeroth order $K K$ mode would then be super partnered with a gravitino, and then Equation (25), assuming that Gravitinos would not last long, would be mapped into the invariance relationship given by Equation (25). We furthermore state that the electromagnetic energy as given in Equation (20) as put into the electro weak phase transition due to a magnetic field $|\boldsymbol{B}| \sim 10^{22} \mathrm{G} \quad[3]$ at the $E W$ epoch would be crucial in the formulation of Equation (25), i.e. hastening the demise of gravitinos (NOT long lived objects) and the invariance of information in keeping fidelity with respect to the cosmological parameters during cosmological evolution.

\section{Conclusion. Several Invariances, due to Mach's Principle and Its Impact upon Massive Graviton Detection}

The main theme, aside from applying conformal cyclic cosmology in a different way, is Equation (25), as well as re-scaling of Mach's principle. According to Gravititino-Graviton Machian ratio invariance, here $\left.M_{\text {electro-weak }}\right|_{\text {Super-partner }}$ is for gravitinos, and $\left.M_{\text {today }}\right|_{\text {Not-Super-Partner }}$ is for the total mass of all of the gravitons in the present universe. This uses a variant of Mistra's [6] Mach's principle value.

$\frac{\left.G M_{\text {electro-weak }}\right|_{\text {Super-partner }}}{R_{\text {electro-weak }} c^{2}} \approx \frac{\left.G M_{\text {today }}\right|_{\text {Not-Super-Partner }}}{R_{0} c^{2}}$ as having a constant ratio value (36)

The benefits from such rescaling are that the evolution of entropy, as seen in 
using $N$ times m (early universe) to $N$ times $m$ (today) can be written in terms of gravitational physics as to the linkage between super partners, SUSY representation of gravitinos and gravitons.

We can use this Machian relationship to understand the $h_{i j}$ values as influenced by massive gravitons. As read from Kurt Hinterbichler [19], if $r=\sqrt{x_{i} x_{i}}$, and we look at a mass induced $h_{i j}$ suppression factor put in of $\exp (-m \cdot r)$, then if

$$
\begin{gathered}
h_{00}(x)=\frac{2 M}{3 M_{\text {Planck }}} \cdot \frac{\exp (-m \cdot r)}{4 \pi \cdot r} \\
h_{0 i}(x)=0
\end{gathered}
$$

$h_{i j}(x)$

$=\left[\frac{M}{3 M_{\text {Planck }}} \cdot \frac{\exp (-m \cdot r)}{4 \pi \cdot r}\right] \cdot\left(\frac{1+m \cdot r+m^{2} \cdot r^{2}}{m^{2} \cdot r^{2}} \cdot \delta_{i j}-\left[\frac{3+3 m \cdot r+m^{2} \cdot r^{2}}{m^{2} \cdot r^{4}}\right] \cdot x_{i} \cdot x_{j}\right)$

Here, we have that these are solutions to the following equation, as given by [19] [20]

$$
\left(\partial^{2}-m^{2}\right) h_{\mu v}=-\kappa \cdot\left[T_{u v}-\frac{1}{D-1} \cdot\left(\eta_{u v}-\frac{\partial_{\mu} \partial_{v}}{m^{2}}\right) \cdot T\right]
$$

To understand the import of the above equations, and the influence of the Machian hypothesis, for $G W$ and massive Graviton signatures from the electro weak regime, set

$$
\begin{aligned}
& M=10^{50} \times 10^{-27} \mathrm{~g} \equiv 10^{23} \mathrm{~g} \propto 10^{61}-10^{62} \mathrm{eV} \\
& M_{\text {Plank }}=1.22 \times 10^{28} \mathrm{eV}
\end{aligned}
$$

And use the value of the radius of the universe, as given by $r=1.422 \times 10^{27}$ meters, and rather than a super partner Gravitino, use the $m_{\text {massive-graviton }} \sim 10^{-26} \mathrm{eV}$.

We argue that the rigorous application of Mach's principle and Equation (20) and Equation (25) permit $h_{i j}$ to be calculated in ways which a magnetic field 3DSR detector can obtain.

For the sake of completeness, we reference Appendix I for its insights as to Gravitons as far as being analogous as to "Gluons". Appendix II has the purpose of delineating what is in the title, i.e. further considerations as to experimental gravity. Keep in mind that this document is a review of General Relativity and the implications of Mach's principle with future revisions in store.

\section{Acknowledgements}

This work is supported in part by National Nature Science Foundation of China grant No. 11375279.

\section{References}

[1] Sarkar, U. (2008) Particle and Astro Particle Physics. Series in High Energy Physics and Gravitation, Taylor \& Francis, New York. 
[2] Kohri, K., Moroi, T. and Yotsuyanagi, A. (2006) Big-Bang Nucleosynthesis with Unstable Gravitino and Upper Bound on the Reheating Temperature. Physical Review D, 73, Article ID: 123511. http://arxiv.org/abs/hep-ph/0507245 https://doi.org/10.1103/physrevd.73.123511

[3] Giovannini, M. http://ned.ipac.caltech.edu/level5/Sept03/Giovannini/Giovan6_3.html

[4] http://abyss.uoregon.edu/ js/cosmo/lectures/lec20.html

[5] Modanese, G. General Estimate for the Graviton Lifetime. http://arxiv.org/pdf/hep-th/9501123.pdf

[6] Mishra, S. (2012) The Quantum theory of the Big Bang: Effective theory of Quantum Gravity. In: O'Connell, J. and Hale, A., Eds., The Big Bang, Theory, Assumptions and Problems, Nova Book Publishers, New York, 205-228.

[7] Arkani-Hamed, N., Dimopoulos, S. and Dvali, G. (1998) The Hierarchy Problem and New Dimensions at a Millimeter. Physics Letters B, 429, 263-272. arXiv:hep-ph/ 9803315 https://doi.org/10.1016/S0370-2693(98)00466-3

[8] Arkani-Hamed, N., Dimopoulos, S. and Dvali, G. (1999) Phenomenology, Astrophysics and Cosmology of Theories with Submillimeter Dimensions and TeV Scale Quantum Gravity. Physical Review D, 59, Article ID: 086004.

arXiv:hep-ph/9807344

https://doi.org/10.1103/PhysRevD.59.086004

[9] Penrose, R. (2011) Cycles of Time. Alfred Knopf, New York.

[10] Beckwith, A. (2011) How to Use the Cosmological Schwinger Principle for Energy, Entropy, and "atoms of Space-Time" to Create a Thermodynamic Space-Time and Multiverse. Journal of Physics. Conference Series, 306, Article ID: 012064.

http://iopscience.iop.org/1742-6596/306/1/012064

http://iopscience.iop.org/1742-6596/306/1;jsessionid=A05372A78C18D970BF35F40 A9A863B51.c2 https://doi.org/10.1088/1742-6596/306/1/012064

[11] Kolb, E.W. and Turner, M.S. (1991) The Early Universe. Westview Frontiers in physics Series, Volume 69.

[12] Park, D.K., Kim, H. and Tamarayan, S. (2002) Nonvanishing Cosmological Constant of Flat Universe in Brane world Scenarios. Physics Letters B, 535, 5-10. https://doi.org/10.1016/S0370-2693(02)01729-X

[13] Kadlecova, H. (2012) Gravitational Field of Gyratons Propagating on Backgrounds Formed by Direct Product Space-Time's. In: Damour, T. and Jantzen, R., Eds., The Marcel Grossman Meetings of General Relativity Proceedings, World Press Scientific, Singapore, 1899-1901.

[14] Kadlecova, H., Zelinikov, A., Krtous, P. and Podolsky, J. (2009) Physical Review D, 80, Article ID: 024004. https://doi.org/10.1103/PhysRevD.80.024004

[15] Ng, Y.J. (2012) Holographic Quantum Foam. In: Damour, T. and Jantzen, R., Eds., The Marcel Grossman Meetings of General Relativity Proceedings, World Press Scientific, Singapore, 2435-2437. https://doi.org/10.1142/9789814374552_0501

[16] Ng, Y.J. (2008) Spacetime Foam: From Entropy and Holography to Infinite Statistics and Nonlocality. Entropy, 10, 441-461. https://doi.org/10.3390/e10040441

[17] Giovannini, M. (2008) A Primer on the Physics of the Cosmic Microwave Background. World Scientific Press, Singapore. https://doi.org/10.1142/6730

[18] Beckwith, A.W. (2011) Identifying a Kaluza Klein Treatment of a Graviton Permitting a Deceleration Parameter Q (Z) as an Alternative to Standard DE. Journal of 
Cosmology, 13, 1-15. http://journalofcosmology.com/BeckwithGraviton.pdf

[19] Hinterbichler, K. (2011) Theoretical Aspects of Massive Gravity. http://arxiv.org/abs/1105.3735.pdf

[20] http://web.mit.edu/redingtn/www/netadv/Xgravitati.html

[21] Berenstein, D., Correa, D.H. and Vazquez, S.E. (2005) Quantizing Open Spin Chains with Variable Length: An Example from Giant Gravitons. Physical Review Letters, 95, Article ID: 191601. arXiv:hep-th/0502172 https://doi.org/10.1103/PhysRevLett.95.191601

[22] Berenstein, D., Correa, D.H. and Vazquez, S.E. (2007) A Study of Open Strings Ending on Giant Gravitons, Spin Chains and Integrability. http://arxiv.org/pdf/hep-th/0604123v2.pdf

[23] Berenstein, D. and Vazquez, S.E. (2005) Integrable Open Spin Chains from Giant Gravitons. Journal of High Energy Physics, 6, 059. arXiv:hep-th/0501078 https://doi.org/10.1088/1126-6708/2005/06/059

[24] Crowell, L. (2005) Quantum Fluctuations of Space-Time. World Scientific Series in Contemporary Chemical Physics: Volume 25, World Press Scientific, Singapore, Republic of Singapore.

[25] Corda, C. (2009) Interferometric Detection of gravitational Waves: The Definitive Test for General Relativity. International Journal of Modern Physics D, 18, 2275 2282. https://arxiv.org/abs/0905.2502 https://doi.org/10.1142/s0218271809015904

[26] Abbott, B.P., et al. (2016) Observation of Gravitational Waves from a Binary Black Hole Merger. Physical Review Letters, 116, Article ID: 061102. https://doi.org/10.1103/physrevlett.116.061102

[27] Abbott, B. P., et al. (2016) GW151226: Observation of Gravitational Waves from a 22-Solar-Mass Binary Black Hole Coalescence. Physical Review Letters, 116, Article ID: 241103. https://doi.org/10.1103/physrevlett.116.241103

[28] Downes, T.G. and Milburn, G.J. (2011) Optimal Quantum Estimation for Gravitation. http://xxx.lanl.gov/abs/1108.5220

[29] Beckwith, A. (2016) Gedanken Experiment for Refining the Unruh Metric Tensor Uncertainty Principle via Schwarzschild Geometry and Planckian Space-Time with Initial Nonzero Entropy and Applying the Riemannian-Penrose Inequality and Initial Kinetic Energy for a Lower Bound to Graviton Mass (Massive Gravity). Journal of High Energy Physics, Gravitation and Cosmology, 2, 106-124. https://doi.org/10.4236/jhepgc.2016.21012

[30] Avessian, A.K. (2009) Planck's Constant Evolution as a Cosmological Evolution Test for the Early Universe. Gravitation and Cosmology, 15, 10-12. https://doi.org/10.1134/S0202289309010034

[31] Goldhaber, A. and Nieto, M. (2010) Photon and Graviton Mass Limits. Reviews of Modern Physics, 82, 939-979. http://arxiv.org/abs/0809.1003http://dx.doi.org/10.1103/RevModPhys.82.939 https://doi.org/10.1103/RevModPhys.82.939 


\section{Appendix I}

Graviton mass problem re-stated in terms of gravitons in terms of chains of gluons. i.e. to look at what happens if we are examining if we extend our analysis of [21] to try to understand the full spectrum of the variable length spin Chain model. This spin chain model has a Hamiltonian given by [22]

$$
\begin{aligned}
H_{\text {Spin-Chain-Model }}= & 2 \lambda \sum_{l=1}^{L} \hat{a}_{l}^{\dagger} \hat{a}_{l}-\lambda \sum_{l=1}^{L-1}\left(\hat{a}_{l}^{\dagger} \hat{a}_{l+1}+\hat{a}_{l} \hat{a}_{l+1}^{\dagger}\right)+2 \lambda \alpha^{2} \\
& +\lambda \alpha \cdot\left(\hat{a}_{1}^{\dagger}+\hat{a}_{1}\right)+\lambda \alpha \cdot\left(\hat{a}_{L}^{\dagger}+\hat{a}_{L}\right)
\end{aligned}
$$

Here

$$
\begin{aligned}
& \hat{a}_{l}^{\dagger} a_{l}=I-|0\rangle\langle 0| \\
& \hat{a}_{l} \hat{a}_{l}^{\dagger}=I
\end{aligned}
$$

The idea is to note, as quoted in the article "The one loop spectrum of anomalous dimensions for strings attached to a maximal giant graviton was described in [23], were it was found that the one loop planar anomalous (given) dimensions correspond to an ordinary spin chain model with integrable Dirichlet-like boundary conditions. This work was extended to study what spin chain corresponds to a more general giant graviton in [21], where we found that the spin chain in question has a variable number of sites and therefore it is not an ordinary spin chain model anymore. After a bosonization transformation, we found that the spin chain model could be also understood in terms of a system of a Cuntz oscillator chain model (a boson chain, where each spin corresponds to a single boson Fock space) with non-diagonal boundary conditions". i.e. one has then, eventually, that there is also a further mathematics generalization [24]. As written by Crowell:

$I$ wrote a paper on $f(R)$ gravity with a massive graviton sector. The graviton can become massive under various (given) circumstances-in theory of course. The scalar curvature in the Hilbert-Palatini action has in a string theory sense a modification as

$$
L=R+\alpha^{\prime} R^{\{a b c d\}} R_{\{a b c d\}}+O\left(\alpha^{\prime 2}\right)
$$

which defines a mass-gap for the graviton. This may have played some role in the early universe, in particular during the inflationary phase. An article by Bern, Dixon and Kosower appeared in Scientific American that is interesting. I read a paper in 2010 or so by the first two authors on computing graviton propagators up to 7 loops. These guys are making a bit of news with these developments (according to L. Crowell)

The article in SciAm talks about gravitons as pair of gluons. This makes in a string theory sense. For a closed string there are two sets of mode operators $a^{\wedge} t_{-} n, a_{-} n$ and $b^{\wedge} t_{-} n, b_{-} n$ for modes propagating left and right polarized directions in space. Along the string though modes travel along a $\sigma$ and $-\sigma$ direction on the string according to whether the $n$ subscript is positive or negative. We then write these modes as $a^{\wedge} f_{-} n, a_{-} n$ and $a^{\wedge} t_{-}\{-n\}, a_{-}\{-n\}$ (ditto for $b$ op- 
erators), and we ignore the zero mode for technical reasons. There is a result which says the Hamiltonian operator must have equal levels in operator products, such as $a^{\wedge} t_{-} n a^{\wedge} t_{-}\{-n\}$, that act on the string ground state. The reason for this is there is no preferred direction along the string with parameter $\sigma$, and this level matching result is a Noether theorem result from this. Each $a^{\wedge} t_{-} n$ or $b^{\wedge} f_{-} n$ is a raising operator for a spin 1 boson field, and the product of the two is a spin 2 field, with no $m=0$ or 1 component. So the graviton can be thought of as a pair of Yang-Mills gauge bosons.. The operators can be given a spacetime index $\mu$ so that we have $\left(a^{\wedge} \mu\right) \wedge t_{-} n$ and $\left(a^{\wedge} \mu\right) \wedge t_{-}\{-n\}$. We then consider this index extended to $\mu=\{0,1,2,3\}$ for spacetime and $q=\{4,5, \ldots, 9\}$. (As a consequence one then finds) A gauge boson operator in standard QFT is then of the form

$$
\left(A^{\mu}\right)^{\dagger}=\left(a^{\mu}\right)_{n}^{\dagger}\left(a^{q}\right)_{-n}^{\dagger}
$$

Suppose we have a gauge boson operator (as written up by L. Crowell is then) of the form

$$
\left(A^{\prime \mu}\right)^{\dagger}=\left(a^{q}\right)_{n}^{\dagger}\left(a^{\mu}\right)_{-n}^{\dagger}
$$

The interaction (as given by L. Crowell) of the two is of the form $\left(A^{\mu}\right)^{\dagger}$ $\left(A^{\prime \mu}\right)^{\dagger}$ and this is then

$$
\left(A^{\mu}\right)^{\dagger}\left(A^{\prime \mu}\right)^{\dagger}=\left(a^{\mu}\right)_{n}^{\dagger}\left(a^{q}\right)_{-n}^{\dagger}\left(a^{q}\right)_{n}^{\dagger}\left(a^{\mu}\right)_{-n}^{\dagger}
$$

Graphically one has that http://f1602.mail.yahoo.com/ya/download?mid=2\%5f0\%5f0\%5f1\%5f51009\%5fA HLai2IAAU4jT5ZSdgFM1RGPHm0\&pid=1.2.2\&fid=Inbox\&inline=1\&appid=Y ahooMailClassic

where the red part that involves the creation of internal space bosons with opposite mode directions on the string. This is equivalent to opposite gauge charges (opposite colors) and so this is a type of glueball, and the annihilation of the opposite charges leaves a product of two operators which recovers a graviton, or two photons.

This is a way of looking at how gravitation is a form of QCD, or that gluon chains are equivalent to a graviton. The diagram in the paper by Bern, Dixon and Kosower of the form below depicts the graviton as a pair of gluons, and in general a gluon chain on the boundary of an anti-de Sitter spacetime has the same symmetries as a graviton in the interior of an anti-de Sitter spacetime The graviton with a mass gap for a spin $s=2$, then has $m=2,1,0,-1,-2$, where the 0 states are the dilaton and axion. The $s=1,-1$ state then corresponds to a massive form of the graviton which may then have a form

$$
\left(A^{\prime \mu}\right)^{\dagger}=\left(a^{q}\right)_{n}^{\dagger}\left(a^{\mu}\right)_{-n}^{\dagger}
$$

(as written above) which may be a massive gauge boson, such as the $Z$ and $W \wedge\{+/-\}$ particle. The axion particle is the gadget which involves $Q C D$ and takes up the $C P$ violation of $Q C D$-leaving $Q C D C P$ symmetric. This might also 
form a component of dark matter as well.

\section{Appendix II}

\section{Further considerations as to experimental gravity. First Inteferometric tests} of General Relativity and the implications of Mach's principle

In doing this, we should keep in mind that what Corda brought up in [25] needs to be looked out, i.e. the interferometric tests of general relativity would be an outgrowth of such investigations.

Furthermore, [26] and [27] should be kept in mind in terms of experimental constraints. Gravitational waves have been discovered, and it is opportune for us to keep [26] and [27] in mind when considering the applications of Equation (B1) below to whatever forms of data sets which may be achievable via experimental gravity. Here Equation (B1) below is a living outgrowth of using [6] with the results that we have

$$
\frac{\left.G M_{\text {electro-weak }}\right|_{\text {Super-partner }}}{R_{\text {electro-weak }} c^{2}} \approx \frac{\left.G M_{\text {today }}\right|_{\text {Not-Super-Partner }}}{R_{0} c^{2}}
$$

Last but not least, the author has already had his own version of Equation (B2), which is given in [28]

$$
\left\langle\left(\delta g_{u v}\right)^{2}\left(\hat{T}^{u v}\right)^{2}\right\rangle \geq \frac{\hbar^{2}}{V_{\mathrm{vol}}^{2}}
$$

The version the author writes is given as seen in [29]. It remains to be seen if [29] is in line with the data sets we may be able to obtain, as well as fidelity with procedures which may allow the issues given in [30] to be thoroughly looked at from an experimental stand point, as well as [31] for the mass of a graviton.

Submit or recommend next manuscript to SCIRP and we will provide best service for you:

Accepting pre-submission inquiries through Email, Facebook, LinkedIn, Twitter, etc. A wide selection of journals (inclusive of 9 subjects, more than 200 journals)

Providing 24-hour high-quality service

User-friendly online submission system

Fair and swift peer-review system

Efficient typesetting and proofreading procedure

Display of the result of downloads and visits, as well as the number of cited articles

Maximum dissemination of your research work

Submit your manuscript at: http://papersubmission.scirp.org/

Or contact jhepgc@scirp.org 